\title{
Infecção Urinária na Gravidez: Análise dos Métodos para Diagnóstico e do Tratamento
}

\author{
Urinary Infection in Pregnancy: Analysis of Diagnostic Methods and Treatment
}

Geraldo Duarte, Alessandra Cristina Marcolin, Carla Vitola Gonçalves, Silvana Maria Quintana, Aderson Tadeu Berezowski, Antônio Alberto Nogueira, Sérgio Pereira da Cunha

\section{RESUMO}

Objetivos: avaliar os aspectos diagnósticos, terapêuticos e as complicações dos casos de infecção do trato urinário (ITU) sintomática durante a gestação, que necessitaram de internação hospitalar.

Métodos: foram incluidas 136 grávidas com diagnóstico clínico de pielonefrite. Foram avaliados: a idade e paridade da paciente, idade gestacional em que foi feito o diagnóstico, antecedentes de importância epidemiológica, propedêutica laboratorial para avaliação da infecção urinária, tratamento e evolução clínica, antimicrobianoprofilaxia e complicações.

Resultados: pielonefrite foi diagnosticada, nas mesmas proporções, em todas as idades gestacionais. Houve maior incidência de ITU entre as primigestas. Apenas 29,3\% das gestantes apresentaram história prévia de ITU. Observou-se que 57,0\% das pacientes apresentaram anemia e 93,0\% mostraram análise urinária alterada. A Escherichia coli foi o uropatógeno mais prevalente (75,8\% dos casos), com baixos percentuais de sensibilidade à ampicilina $(60,6 \%)$ e à cefalotina $(63,6 \%)$ e altos percentuais de sensibilidade à cefuroxima (95,5\%). A maior taxa de melhora clinica foi obtida entre as gestantes tratadas com cefuroxima (95,7\%). A antimicrobianoprofilaxia foi necessária em 11,0\% das pacientes. $O$ trabalho de parto pré-termo ocorreu em 33,3\% das gestantes que deram à luz em nosso serviço, e o parto pré-termo em 18,9\%.

Conclusões: esses resultados reforçam a necessidade do diagnóstico precoce e tratamento efetivo da ITU em gestantes, a fim de evitar a ocorrência freqüente de complicações perinatais, como o trabalho de parto e o parto pré-termo. Destaca-se a necessidade de avaliação periódica do padrão de sensibilidade dos agentes etiológicos prevalentes aos antimicrobianos de uso permitido durante a gestação, adotando-se a cefuroxima como o antimicrobiano de escolha para o tratamento das ITU na gestação.

PALAVRAS-CHAVE: Infecção urinária. Pré-natal. Complicações da gravidez.

\section{Introdução}

A infecção do trato urinário representa uma das doenças infecciosas mais comuns durante a gestação, com freqüência variando de 5 a $10 \%{ }^{1,2}$.

Departamento de Ginecologia e Obstetrícia do Hospital das Clínicas da Faculdade de Medicina de Ribeirão Preto Universidade de São Paulo

Correspondência:

Geraldo Duarte

Avenida Bandeirantes, 3900

Hospital das Clínicas da Faculdade de Medicina de Ribeirão

Preto - USP

14049-900 - Ribeirão Preto - SP

Fone: (16) 602-2588

e-mail: gduarte@fmrp.usp.br
Essa infecção pode ser sintomática ou assintomática, notando-se na gravidez a ocorrência de fatores que facilitam a mudança de infecções assintomáticas para sintomáticas ${ }^{3}$. Além da incidência aumentada dessas infecções entre grávidas, é justamente neste período que o arsenal terapêtico antimicrobiano e as possibilidades profiláticas são mais restritas, considerando-se a toxicidade das drogas para o feto.

As transformações anatômicas e fisiológicas que ocorrem no trato urinário durante a gravidez facilitam o desenvolvimento de infecções urinárias sintomáticas em mulheres, que muitas vezes já apresentam bacteriúria no momento da concepção ${ }^{4}$. A compressão extrínseca dos ureteres e a redução 
da atividade peristáltica provocada pela progesterona provocam dilatação progressiva das pelves renais e ureteres. Essas mudanças, junto com o aumento do débito urinário, levam à estase urinária. A estase ainda é favorecida pela diminuição do tônus vesical, com subseqüente aumento da capacidade da bexiga e seu esvaziamento incompleto, facilitando o refluxo vesicoureteral e pielonefrites. Além disso, o rim perde sua capacidade máxima de concentrar a urina, reduzindo sua atividade antibacteriana, e passa a excretar quantidades maiores de glicose e aminoácidos, fornecendo meio apropriado para a proliferação bacteriana ${ }^{3,5-7}$.

A urolitíase também pode facilitar a ocorrência de quadros de infecção do trato urinário durante a gravidez, sendo causa freqüente de dor e internação nesse período. Cerca de 0,026-1,14\% das gestações são complicadas por litíase urinária ${ }^{8}$.

O perfil microbiológico das infecções urinárias na gravidez é bem conhecido. A Escherichia coli é o uropatógeno mais comum, sendo responsável por mais de três quartos dos casos. Por isso, a terapêtica inicial necessariamente deve levar em consideração o padrão de sensibilidade desse microrganismo aos antimicrobianos propostos ${ }^{9}$.

A associação entre infecção do trato urinário e a piora do prognóstico gestacional é conhecida há muito tempo. Dentre as complicações destacam-se o trabalho de parto e parto pré-termo, ruptura prematura de membranas amnióticas, restrição de crescimento intra-útero, recém-nascidos de baixo peso e óbito perinatal ${ }^{10-12}$. Gestações complicadas por infecção urinária estão associadas ao dobro da mortalidade fetal observada em gestações normais de uma mesma área geográfi$\mathrm{ca}^{3}$. Outras complicações da gravidez têm sido associadas às infecções urinárias, incluindo-se a hipertensão e a pré-eclâmpsia ${ }^{13}$, anemia ${ }^{14}$, corioamnionite, endometrite e septicemias. Porém, o que não se sabe com certeza é se o episódio de infecção urinária precede a ocorrência dessas complicações ou se essas já existiam no momento do diagnóstico da infecção do trato urinário ${ }^{3,11}$.

Após o diagnóstico clínico da infecção urinária aguda e confirmação com exame de urina tipo I, na maioria dos casos a instituição do tratamento demanda urgência, sem tempo para a obtenção do resultado do urocultivo e antibiograma. Este fato torna imprescindivel a avaliação periódica do perfil microbiológico e da sensibilidade dos agentes etiológicos mais prevalentes aos antimicrobianos, em face do crescente aumento de germes resistentes aos poucos antibióticos de uso seguro durante o periodo gestacional.

O risco aumentado de desenvolvimento de infecção urinária sintomática na gestação e a necessidade de se avaliarem periodicamente os aspectos microbiológicos e terapêuticos dos casos de infecção urinária justificam a presente pesquisa.

\section{Pacientes e Métodos}

Foram incluídas neste estudo 136 gestantes internadas na Enfermaria de Gestação de Alto Risco do HCFMRP-USP para tratamento de infecção do trato urinário, no período de janeiro de 1999 a dezembro de 2000. Considerou-se como portadora de infecção do trato urinário toda gestante com diagnóstico clínico inequívoco dessa infecção e que foi medicada para tal, independente do resultado da urocultura obtido posteriormente à sua admissão. Os dados foram coletados, de forma retrospectiva, por meio da análise dos prontuários médicos das pacientes selecionadas. Os critérios de inclusão foram: 1) portadoras de infecção do trato urinário, 2) avaliação laboratorial completa e 3) internação para tratamento.

Todas as pacientes foram submetidas à avaliação laboratorial que inclui hemograma completo, urina tipo I, urocultura e ultra-sonografia de rins e vias urinárias. Os valores normais de referência para hemoglobina e glóbulos brancos, durante a gestação, seguiram as orientações do Manual de Orientação de Assistência Pré-Natal do Ministério da Saúde ${ }^{15}$. Consideraram-se como normais os valores de hemoglobina $\geq 11 \mathrm{~g} / \mathrm{dL}$. Para glóbulos brancos, o limite superior de normalidade aceito, para o período gravídico, foi de 15.000 leucócitos / mL. Na análise do exame de urina tipo I, considerou-se como normal: contagem de leucócitos de até 4 por campo, de hemácias de até 2 por campo, ausência de cristais e cilindros, glicosúria, bacteriúria e nitrito negativos.

Com relação às uroculturas, foram consideradas positivas todas aquelas em que houve crescimento bacteriano, independente do número de colônias. A sensibilidade do microrganismo foi avaliada com relação à concentração urinária da droga testada. Para realização da urocultura e do exame de urina tipo I utilizou-se a urina coletada pela técnica do jato médio, após anti-sepsia perineal. Os exames ultra-sonográficos foram realizados com uso do aparelho de ultra-sonografia da Advanced Technology Laboratories (ATL), modelo HDI-3000 (Bothell-WA, USA), por meio de sonda convexa de 4-2 $\mathrm{MHz}$, após preparo intestinal e bexiga repleta.

Os diversos tratamentos realizados seguiram as normas estabelecidas no "Protocolo de Conduta em Gestação de Alto Risco" em vigor no Departamento de Ginecologia e Obstetrícia do Hospital das Clinicas da Faculdade de Medicina de Ribeirão Preto da Universidade de São Paulo (HCFMRP-USP). O antimicrobiano mais utilizado foi a cefalotina ( 1 grama endovenoso de $6 / 6 \mathrm{~h}$ ) até se obter melhora clínica do quadro, quando então 
foi substituída por cefalexina (500 mg via oral de $6 / 6 \mathrm{~h})$ até o término do tratamento. Outros esquemas terapêuticos utilizados foram baseados em antibiograma ou história prévia de tratamento com cefalotina/cefalexina sem sucesso: cefuroxima (750 $\mathrm{mg}$ endovenosa de $8 / 8 \mathrm{~h}$ ), norfloxacina (400 mg via oral de 12/12 h). Considerou-se como melhora clínica a remissão do quadro clínico agudo, principalmente da febre, dor lombar e sintomas sistêmicos gerais (náuseas, vômitos, malestar).

As principais indicações de profilaxia foram: mais de dois episódios de qualquer forma clínica de infecção urinária durante a gestação; duas pielonefrites ou um episódio de infecção urinária grave associado a um fator de risco (malformação, cálculo ou dilatação pielocalicial). A droga mais utilizada foi a nitrofurantoína (100 mg via oral ao dia).

Foram analisados os seguintes parâmetros: idade e paridade da gestante, idade gestacional do diagnóstico da infecção, antecedentes de importância epidemiológica, propedêutica laboratorial para avaliação da infecção urinária, tratamento e evolução clínica, antimicrobianoprofilaxia e complicações.

Para a análise estatística utilizou-se o teste do $\chi^{2}$ para comparar a incidência de infecção urinária entre as diversas idades gestacionais e entre as pacientes de acordo com sua paridade, com nível de significância de $5 \%$.

O presente estudo foi conduzido após sua aprovação pelo Comitê de Ética em Pesquisa do HCFMRP-USP.

\section{Resultados}

A média de idade das pacientes estudadas foi de 21,8 anos. Nesse grupo de gestantes, 93,2\% dos casos de infecção urinária foram diagnosticados antes do termo da gravidez, havendo distribuição uniforme ao longo das diversas idades gestacionais.

Com relação à paridade, observou-se maior incidência de infecção urinária nas pacientes primigestas $(51,5 \%)$. Foram verificadas diferenças estatisticamente significativas em relação às pacientes com duas ou mais gestações $(p<0,001)$.

A Tabela 1 permite focalizar os antecedentes mórbidos maternos mais importantes para infecção urinária em gestantes. Com relação às condições predisponentes, devem ser salientados os tratamentos prévios de outros episódios de infecção urinária na gestação atual $(19,8 \%)$ e o tratamento, sem sucesso, da infecção atual $(6,6 \%)$. Infecção urinária de repetição (três ou mais episó- dios de infecção urinária ao ano) foi identificada em apenas $2,9 \%$ das pacientes. Cumpre destacar que, em $70,0 \%$ dos casos, nenhum antecedente mórbido importante foi encontrado.

Tabela 1 - Distribuição dos principais antecedentes epidemiológicos das grávidas com infecção do trato urinário.

\begin{tabular}{lcc}
\hline Antecedentes & $\mathbf{n}$ & $\%$ \\
\hline Nenhum & 91 & 70,0 \\
Tratamento prévio de ITU na gestação & 27 & 19,8 \\
ITU atual em tratamento & 09 & 6,6 \\
ITU de repetição & 04 & 2,9 \\
Trabalho de parto pré-termo prévio & 03 & 2,2 \\
Litíase renal & 02 & 1,5 \\
Total & 136 & 100 \\
\hline
\end{tabular}

ITU: infecção do trato urinário.

No momento da admissão hospitalar, as pacientes foram submetidas à avaliação laboratorial. Observou-se dosagem normal de hemoglobina em $43,0 \%$ e anemia moderada $(\mathrm{Hb}<11 \mathrm{~g} / \mathrm{dL}$ e $>8$ $\mathrm{g} / \mathrm{dL}$ ) em $57,0 \%$ das pacientes.

Com relação à contagem de leucócitos no sangue, constatou-se normalidade para o período gravídico em $50,4 \%$ das pacientes. Observou-se que apenas $41,8 \%$ dos leucogramas apresentaram leucócitos com desvio à esquerda (formas jovens circulantes), incluindo-se $29 \%$ dos leucogramas considerados normais pelo número de leucócitos.

Todas as pacientes foram submetidas a exame de urina tipo I. Apenas 7\% dos exames mostraram normalidade. Bacteriúria e nitrito foram encontrados em 93,7 e 58,2\% dos exames analisados, respectivamente. Proteinúria e hematúria foram vistas na grande maioria dos exames $(77,0$ e $64,7 \%$, respectivamente), indicando lesão renal.

Dos 136 casos incluidos no estudo, $93(68,4 \%)$ foram submetidas à exame ecográfico de rins e vias urinárias. Dilatação pielocalicial renal, principalmente à direita, foi encontrada em $51,6 \%$ dos exames realizados. Cálculos e cistos renais foram detectados em $13,0 \%$ e 3,2\% dos exames, respectivamente. Observou-se porcentagem importante de exames normais: 32,3\%.

Das 136 uroculturas colhidas no momento da internação, 87 foram positivas $(64,0 \%)$. Os principais microrganismos identificados nessas uroculturas estão listados na Tabela 2, assim como a sensibilidade das cepas encontradas aos diversos antimicrobianos testados. Destaca-se, pela sua freqüência, a Escherichia coli em $75,8 \%$ dos casos. Estas cepas apresentaram sensibilidade aos antibióticos mais freqüentemente empregados: 95,5\% para a cefuroxima a $100 \%$ para a cefafolina. 
Tabela 2 - Distribuição dos microrganismos mais freqüentemente isolados em uroculturas de gestantes com infecção urinária e o percentual de sensibilidade das cepas aos principais antimicrobianos testados.

\begin{tabular}{|c|c|c|c|c|c|c|c|c|c|c|c|c|c|c|}
\hline Micro & $\mathrm{n}$ & $\%$ & $\begin{array}{c}\text { A.nalid } \\
\%\end{array}$ & $\begin{array}{c}\text { Nitrof } \\
\%\end{array}$ & $\begin{array}{c}\text { Ampi } \\
\%\end{array}$ & $\begin{array}{c}\text { Cefal } \\
\%\end{array}$ & $\begin{array}{c}\text { Cipro } \\
\%\end{array}$ & $\begin{array}{c}\text { Norflox } \\
\%\end{array}$ & $\begin{array}{c}\text { Amica } \\
\%\end{array}$ & $\begin{array}{c}\text { Sulfa } \\
\%\end{array}$ & $\begin{array}{c}\text { Cxm } \\
\%\end{array}$ & $\begin{array}{c}\mathrm{Cfz} \\
\%\end{array}$ & $\begin{array}{c}\text { Cefox } \\
\%\end{array}$ & $\begin{array}{c}\text { Genta } \\
\%\end{array}$ \\
\hline E. coli & 66 & 75,8 & 98,5 & 93,9 & 60,6 & 63,6 & 97,0 & 98,5 & 100 & 71,2 & 95,5 & 100 & 97,0 & 8,5 \\
\hline K. pneumoniae & 08 & 9,1 & 100 & 75,0 & 100 & 75,0 & 100 & 100 & 100 & 87,5 & 100 & 100 & 100 & 100 \\
\hline Strepto agalactiae & 04 & 4,5 & 100 & 100 & 100 & 75,0 & 100 & 100 & 100 & 100 & 75,0 & 100 & 100 & 100 \\
\hline Proteus mirabilis & 03 & 3,4 & 100 & 0,0 & 100 & 100 & 100 & 100 & 100 & 66,7 & 100 & 100 & 100 & 66,7 \\
\hline Enterobacter sp & 02 & 2,4 & 100 & 0,0 & 0,0 & 0,0 & 100 & 100 & 100 & 50,0 & 50,0 & 50,0 & 0,0 & 100 \\
\hline Outros & 04 & 4,8 & 100 & 75,0 & 25,0 & 75,0 & 100 & 100 & 100 & 75,0 & 75,0 & 75,0 & 100 & 100 \\
\hline
\end{tabular}

$\mathrm{n}=$ número de uroculturas positivas

A. nalid = Ácido Nalidíxico; Nitrof = Nitrofurantoína; Ampi = Ampicilina; Cefal = Cefalotina; Cipro = Ciprofloxacina; Norflox = Norfloxacina; Amica = Amicacina; Sulfa = Sulfametoxazol Trimetoprima; Cxm = Cefuroxima; $\mathrm{Cfz}=$ Ceftazidima; Cefox = Cefoxitina; Genta = Gentamicina

Após instituição do antimicrobiano para tratamento hospitalar da infecção urinária, observouse melhora clínica em $91,9 \%$ dos casos, destacando-se que a maior porcentagem de melhora ocorreu no grupo de pacientes usuárias de cefuroxima (Tabela 3).

Tabela 3 - Distribuição dos antibióticos utilizados para o tratamento de infecção urinária em 136 gestantes e a evolução clínica.

\begin{tabular}{lrrrrrrr}
\hline & \multicolumn{4}{c}{ Melhora clínica } & \multicolumn{2}{c}{} \\
Antibióticos & \multicolumn{2}{c}{ Sim } & \multicolumn{2}{c}{ Não } & \multicolumn{2}{c}{ Total } \\
& $\mathbf{n}$ & \%total $^{*}$ & $\mathbf{n}$ & \%total $^{*}$ & \multicolumn{1}{c}{$\mathbf{n}$} & \%136 $^{+}$ \\
\hline Cefalotina & 83 & 91,2 & 08 & 8,8 & 91 & 66,9 \\
Cefuroxima & 22 & 95,7 & 01 & 4,3 & 23 & 16,9 \\
Norfloxacina & 11 & 84,6 & 02 & 15,4 & 13 & 9,6 \\
Cefalexina & 05 & 100,0 & 0 & 0 & 05 & 3,7 \\
Ceftriaxona & 02 & 100,0 & 0 & 0 & 02 & 1,5 \\
Nitrofurantoína & 01 & 100,0 & 0 & 0 & 01 & 0,7 \\
Ampicilina & 01 & 100,0 & 0 & 0 & 01 & 0,7 \\
Total & 125 & 91,9 & 11 & 8,1 & 136 & 100,0
\end{tabular}

*\%/total: \% de pacientes que melhoraram (ou não) usando um dado antibiótico;

+ \% 136: porcentagem de pacientes que usaram um dado antibiótico no total de gestantes estudadas.

Para as 11 pacientes que não apresentaram melhora do quadro clínico agudo $(8,1 \%)$, foi efetuada troca do antibiótico, baseada no resultado do antibiograma, da seguinte forma: oito usuárias de cefalotina passaram a receber cefuroxima (1), norfloxacina (6) e ampicilina (1); duas usavam norfloxacina e passaram a receber cefalexina (1) e ceftriaxona (1) e uma paciente que usava cefuroxima passou a utilizar ceftriaxona. Todas essas pacientes obtiveram melhora clínica após a troca do antibiótico.

As complicações obstétricas estão listadas na Tabela 4. Nota-se que a principal complicação associada à infecção urinária foi o trabalho de parto pré-termo. Com relação à utilização de profilaxia, $11,0 \%$ das pacientes fizeram uso de antibioticoprofilaxia para infecção urinária.

Tabela 4 - Complicações materno-fetais ocorridas no grupo de gestantes com infecção urinária.

\begin{tabular}{llc}
\hline Complicações & $\mathbf{n}$ & \%* \\
\hline Trabalho de parto pré-termo & 30 & 33,3 \\
Parto pré-termo & 17 & 18,9 \\
Óbito fetal & 02 & 2,2 \\
Hepatite transinfecciosa & 01 & 1,1 \\
Outros & 03 & 3,3 \\
\hline
\end{tabular}

* \% de complicações pelo total de pacientes que tiveram a gestação resolvida no HCFMRP-USP

\section{Discussão}

A freqüência e a gravidade das infecções urinárias durante a gravidez têm sido reconhecidas há mais de um século. Além de constituírem problema relativamente comum no período gestacional, muitas questões sobre esse assunto ainda permanecem controversas e tornam-se motivo de investigação clínica ${ }^{3}$. O tema adquire relevância ao notar-se sua associação com piores prognósticos maternos e perinatais ${ }^{11,16,17}$.

As alterações anátomo-funcionais que ocorrem no trato urinário durante a gestação tornamse mais evidentes no segundo e, principalmente, no terceiro trimestre ${ }^{10}$. Com base nesse detalhe epidemiológico, alguns autores justificam que o risco de infecção urinária sintomática aumenta com a idade gestacional ${ }^{5}$. No entanto, na presente casuística, este achado não foi confirmado. Com relação à paridade, os resultados aqui descritos não confirmam a assertiva que relaciona maior incidência de infecção com multiparidade, já bem 
estabelecida na literatura ${ }^{1,3}$. Entretanto, confirmam os achados encontrados por Baleiras et al. ${ }^{5}$, que demonstraram $56 \%$ de nuliparas no grupo de gestantes com infecção urinária.

Em 1999, Pastore et al. ${ }^{18}$ conduziram estudo de coorte formado por 6.795 gestantes em seguimento pré-natal, tentando identificar, na primeira consulta, características epidemiológicas capazes de predizer infecção urinária. Encontraram duas fortes características: história de infecção urinária prévia à gravidez e infecção na gravidez atual antes de iniciar o pré-natal. No presente estudo, cerca de um terço das pacientes enquadraram-se nesses critérios. Por outro lado, dois terços delas não apresentaram qualquer fator de risco para infecção urinária. Logo, embora a história de infecção urinária pregressa seja mais comum nas pacientes com bacteriúria assintomática, não é possivel identificar com precisão a subpopulação de risco apenas por esse critério. Por isso, torna-se necessário submeter toda gestante a exames de triagem para bacteriúria assintomática, a fim de se instituir tratamento precoce e reduzir a taxa de progressão para infecções sintomáticas ${ }^{3,4,10,19,20}$.

$\mathrm{Na}$ avaliação laboratorial das gestantes desse trabalho, encontrou-se elevada taxa de anemia. Apesar de o processo anêmico crônico favorecer o desenvolvimento da infecção, outros fatores devem estar envolvidos na anemia materna. Parece que anemia hemolitica pode surgir em um quarto das gestantes com infecção urinária como conseqüência de dano na membrana plasmática do eritrócito, mediado por endotoxinas bacterianas ${ }^{14}$.

Com relação à avaliação dos leucogramas, observou-se que, em mais da metade deles, a contagem global de leucócitos foi normal para o período gravídico. Talvez o leucograma não seja exame laboratorial essencial no diagnóstico de infecção urinária, mas é de importância fundamental no seguimento da paciente com quadros clínicos mais graves.

Por outro lado, os exames de urina tipo I mostraram-se alterados na quase totalidade dos casos estudados. Porém, não deve ser esquecido que leucocitúria, proteinúria e cilindros são somente sinais de inflamação, não equivalendo a bacteriúria significativa. Atualmente, foram introduzidos testes rápidos e de baixo custo para o diagnóstico de infecção urinária, baseados no método colorimétrico. Dois desses testes se revestem de importância. O teste do nitrito, com sensibilidade de $50 \%$ e especificidade de $97-100 \%$, pode ter resultados falso-positivos, quando utilizado em urina contaminada por germes vaginais ou muito concentrada. O teste da esterase de leucócitos (reflete leucocitúria) possui baixa sensibilidade e es- pecificidade $(25 \%)$ e também pode apresentar resultados falso-positivos em casos de pacientes com infecções vaginais ${ }^{21}$. Ambos os testes apresentam baixa sensibilidade e, portanto, não servem como teste para rastreamento. A urocultura, portanto, ainda é o padrão-ouro para o diagnóstico laboratorial de infecção urinária ${ }^{10}$.

A ecografia dos rins e vias urinárias é um exame complementar importante nos casos de infecção urinária, além de ser rápido, barato e totalmente inócuo para a mãe e para o feto. Pode fornecer informações sobre fatores predisponentes, como cálculos urinários e dilatação do sistema coletor $\mathrm{renal}^{8}$. Mais de $80 \%$ das gestantes apresentam dilatação pielocalicial secundária às mudanças hormonais e anatômicas que ocorrem na gestação, sendo o rim direito o mais afetado e principalmente no terceiro trimestre ${ }^{22}$. Esses achados são compativeis com os do presente estudo.

Nas 136 uroculturas realizadas, observouse elevado índice de uroculturas negativas $(36,0 \%)$ em pacientes com sinais clinicos inequívocos de infecção urinária. Este fato obriga relembrar algumas intercorrências no processamento do material coletado, como o armazenamento por tempo excessivo em condições inadequadas e precárias técnicas laboratoriais. Ainda, atenção deve ser dada à colheita adequada de urina, dando-se ênfase à limpeza perineal satisfatória e à técnica do jato médio ${ }^{10}$. Do ponto de vista laboratorial, é aceitável que até $5,0 \%$ das uroculturas apresentem resultados negativos, cifra bem menor que os indices de nosso trabalho. Infelizmente, esses resultados falso-negativos interferem na escolha do melhor tratamento, baseado em antibiogramas ${ }^{9}$.

No presente trabalho, o principal uropatógeno isolado foi a Escherichia coli. Outros organismos como Klebsiella pneumoniae, Proteus mirabilis, espécies de Staphylococcus, Streptococcus e enterobactérias contribuem para a grande maioria dos casos restantes. Essa distribuição é totalmente compativel com a literatura consulta$\mathrm{da}^{3,5,6,10,23}$.

Avaliando o padrão de sensibilidade das bactérias isoladas nas uroculturas, verificou-se que a maioria delas apresenta altos índices de sensibilidade a antimicrobianos como a cefuroxima, ceftazidima, cefoxitina, nitrofurantoina, aminoglicosídeos e quinolonas. Por outro lado, os percentuais de sensibilidade dos microrganismos isolados, principalmente da Escherichia coli, a ampicilina, sulfa e cefalotina estão longe dos ideais. Esse resultado é preocupante, uma vez que a cefalotina e a ampicilina ainda são bastante utilizadas no tratamento das infecções urinárias, em decorrência do baixo custo relativo. No HCFMRPUSP, a cefalotina foi, por muitos anos, o antibióti- 
co mais utilizado no tratamento da pielonefrite em gestantes, e os achados deste trabalho levou-nos a excluir essa droga como primeira escolha. Atualmente, a cefuroxima é o antimicrobiano mais utilizado para o tratamento das infecções urinárias em gestantes.

A escolha de um antimicrobiano deve levar em conta, além da sensibilidade das bactérias mais prevalentes, outros fatores, tais como: a facilidade de obtenção pela paciente, a sua tolerabilidade, a comodidade do esquema posológico, seu custo e sua toxicidade ${ }^{3,5,6,9}$.

A associação de infecção urinária com uma grande variedade de complicações maternas e perinatais já foi bem documentada ${ }^{10-12}$. Os parâmetros avaliados nessa casuística permitem dizer que o trabalho de parto pré-termo foi a principal complicação encontrada. McKenzie et al. ${ }^{24}$ observaram aumento da incidência de trabalho de parto e parto pré-termo em gestantes com anticorpos urinários elevados para antígenos de Escherichia coli. O mesmo fato foi notado quando os anticorpos para antígenos do Streptococcus do grupo B se mostraram elevados na urina dessas pacientes. Logo, o início do trabalho de parto pode ser devido à resposta inflamatória local, secundária às infecções urogenitais. Outro mecanismo pelo qual o trabalho de parto pode ser desencadeado seria a colonização do fluido amniótico por bactérias vindas do foco infeccioso urinário, produtoras de fosfolipases e, em última análise, de prostaglandinas $^{11,25}$.

Para se reduzir as taxas de infecção urinária e suas complicações durante a gravidez, várias etapas devem ser consideradas, em diversos pontos da assistência obstétrica: solicitar urocultura precocemente no pré-natal, para diagnosticar e tratar os casos de bacteriúria assintomática; utilizar o tratamento antimicrobiano mais eficaz; propiciar seguimento em pré-natal de alto risco e garantir o tratamento das complicações maternas e perinatais, em hospital com condições adequadas para isso.

\section{ABSTRACT}

Purpose: to assess the diagnostic and therapeutic aspects and the complications of symptomatic urinary tract infections (UTI) during pregnancy of patients who were hospitalized. Methods: a total of 136 pregnant women with a clinical diagnosis of pyelonephritis were studied. The studied parameters were: age and parity of patients, gestational age of diagnosis, epidemiologic aspects, laboratory evaluation for UTI, treatment and clinic evolution, prophylaxis and complications.

Results: pyelonephritis was diagnosed at the same proportions at all gestational ages. The incidence of UTI was higher among primigravidae. Only 29.3\% of the pregnant women had a previous history of UTI; $57.0 \%$ were anemic and 93.0\% had altered urinalysis. Escherichia coli was the most prevalent uropathogen (75.8\% of cases), with low percentages of sensitivity to ampicillin (60.6\%) and high percentages of sensitivity to cefuroxime $(95,5 \%)$. The highest rate of clinical improvement was obtained for the pregnant women treated with cefuroxime (95.7\%). Prophylaxis was needed in $11.0 \%$ of the patients. Preterm labor occurred in $33.3 \%$ of the pregnant women who delivered in our service and preterm delivery occurred in $18.9 \%$.

Conclusions: the present results support the need for an early diagnosis and effective treatment of UTI in pregnant women in order to prevent the frequent occurrence of perinatal complications such as premature labor and delivery. We emphasize the need of a periodical evaluation of the pattern of sensitivity of the etiologic agents to the antimicrobials allowed for use during pregnancy, with cefuroxime being adopted as the antibiotic of choice for the treatment of UTI during pregnancy.

KEY WORDS: Urinary infection. Prenatal care. Complications of pregnancy.

\section{Referências}

1. Andriole VT. Urinary tract infections in the 90s: pathogenesis and management. Infection 1992; 20 (Suppl 4):S251-6.

2. Delcroix M, Zone V, Cheront C, Adam MH, Duquesne G, Noel AM. L'infection urinaire de la femme enceinte. Rev Fr Gynécol Obstét 1994; 89:277-84.

3. Andriole VT, Patterson TF. Epidemiology, natural history, and management of urinary tract infections in pregnancy. Med Clin North Am 1991; 75:359-73.

4. Stenqvist K, Dahlen-Nilsson I, Lidin-Janson G, et al. Bacteriuria in pregnancy. Frequency and risk of acquisition. Am J Epidemiol 1989; 129:372-9.

5. Baleiras C, Campos A, Lourenço I, Revez AI. Infecções urinárias e gravidez. Acta Med Port 1998; 11:839-46.

6. Mikhail MS, Anyaegbunam A. Lower urinary tract dysfunction in pregnancy: a review. Obstet Gynecol Survey 1995; 50:675-83.

7. Lucas MJ, Cunningham FG. Urinary infection in pregnancy. Clin Obstet Gynecol 1993; 36:855-68.

8. Swanson SK, Heilman RL, Eversman WG. Urinary tract stones in pregnancy. Surg Clin North Am 1995; 75:123-42. 
9. Duarte G, Matos MA, Cunha SP, Nogueira AA, Mauad Filho F, Berezowski AT. Infecção urinária durante a gravidez. Rev Bras Ginecol Obstet 1997; 19:495-503.

10. Millar LK, Cox SM. Urinary tract infections complicating pregnancy. Infect Dis Clin North Am 1997; 11:13-26.

11.Schieve LA, Handler A, Hershow R, Persky V, Davis F. Urinary tract infection during pregnancy: its association with maternal morbidity and perinatal outcome. Am J Public Health 1994; 84:405-10.

12.Duarte G, Cunha SP, Mauad Filho F, Berezowski AT, Baruffi I. Feto morto. I. Aspectos conceituais e etiopatogênicos (análise de 437 casos). Rev Bras Ginecol Obstet 1985; 7:115-8.

13.Lee CJ, Hsieh TT, Chiu TH, Chen KC, Lo LM, Hung TH. Risk factors for pre-eclampsia in an Asian population. Int J Gynaecol Obstet 2000; 70:327-33.

14.Cox SM, Shelburne P, Mason R, Guss S, Cunningham FG. Mechanisms of hemolysis and anemia associated with acute antepartum pyelonephritis. Am J Obstet Gynecol 1991; 164:587-90.

15.Amed AM, Silva Filho AR, Alencar Júnior CA, et al. Modificações fisiológicas da gravidez. In: Alencar Júnior CA, editor. Assistência Pré-natal: manual de orientação. $1^{\text {a }}$ ed. São Paulo: Federação Brasileira das Sociedades de Ginecologia e Obstetrícia; 2000. p.16-24.

16.Cunningham FG, Lucas MJ. Urinary tract infections complicating pregnancy. Baillieres Clin Obstet Gynaecol 1994; 8:353-73.

17.Parrillo JE. Pathogenic mechanisms of septic shock. N Engl J Med 1993; 328:1471-7.
18.Pastore LM, Savitz DA, Thorp JM Jr. Predictors of urinary tract infection at the first prenatal visit. Epidemiology 1999; 10:282-7.

19.Patterson TF, Andriole VT. Detection, significance, and therapy of bacteriuria in pregnancy. Update in the managed health care era. Infect Dis Clin North Am 1997; 11:593-608.

20.Gratacós E, Torres PJ, Vila J, Alonso PL, Cararach V. Screening and treatment of asymptomatic bacteriuria in pregnancy prevent pyelonephritis. J Infect Dis 1994; 169:1390-2.

21.Olsen BE, Hinderaker SG, Lie RT, et al. The diagnosis of urinary tract infections among pregnant women in rural Tanzania; prevalences and correspondence between different diagnostic methods. Acta Obstet Gynecol Scand 2000; 79:729-36.

22. Croce P, Signorelli P, Chiapparini I, Dede A. Hydronephrosis in pregnancy. Ultrasonographic study. Minerva Ginecol 1994; 46:147-53.

23. Millar LK, Wing DA, Paul RH, Grimes DA. Outpatient treatment of pyelonephritis in pregnancy: a randomized controlled trial. Obstet Gynecol 1995; 86:560-4.

24.McKenzie H, Donnet ML, Howie PW, Patel NB, Benvie DT. Risk of preterm delivery in pregnant women with group B streptococcal urinary infections or urinary antibodies to group $B$ streptococcal and E. Coli antigens. Br J Obstet Gynaecol 1994; 101:107-13.

25. Câmara PAD. Infecção urinária na gravidez. In: Cunha SP, Duarte G, editores. Gestação de Alto Risco. $1^{\text {a }}$ ed. Rio de Janeiro: MEDSI; 1998. p.211-20.

\section{RBGO}

\section{é uma publicação da FEBRASGO}

\section{que aceita artigos provenientes de ginecologistas,} obstetras e de outras especialidades. Portanto, publique!!! 